\title{
Experimental Research on Corrosion-Induced Cracking Monitoring Based on Optical Fiber Sensor
}

\author{
Mao Jianghong, Jin Weiliang, Xiong Yanjun, and Wang Qi \\ Ningbo Institute of Technology, Zhejiang University \\ He Yong Zhang Jun \\ Institute of Structural Engineering, Zhejiang University
}

\begin{abstract}
Corrosion-induced cracking is a widely existent issue for coastal infrastructure, which leads to its advanced failure. The monitoring of corrosion-induced cracking is an important means of evaluating its influence on structure normal use and safe operation. However, traditional sensors such as strain gage are unsuitable to embed into concrete and to record long-term strain of concrete caused by steel bar corrosion. The optical fiber sensor, Brillouin Optical Time Domain Analysis (BOTDA) can effectively avoid the undetected phenomenon existing in point-wise test method, and it has the characteristic of automated monitoring. The optical fiber sensor is also electrical insulation and anti-electromagnetic interference; so it is suitable for detecting the corrosion-induced cracks. In this article, experimental research on corrosion-induced crack monitoring based on BOTDA is introduced. The distributed optical fiber is embedded into concrete around the steel bar to record expansion force of concrete. An accelerated corrosion test is performed to investigate the relationship between tiny geometrical changes of steel bar and concrete expansion force. Different rates of corrosion current are applied to the specimen. The accelerated corrosion test approved that the optical fiber sensor can effectively monitor the whole process of corrosion-induced cracking.
\end{abstract}

\section{INTRODUCTION}

Reinforcement corrosion has been considered as one of the major causes for the deterioration of RC structures (Tuutti, 1982). The corrosion of steel bar would lead to obvious volume expansion. The expansive force acting on concrete could result in cracking, spalling, and delamination. (Alonso, Andrade, Rodriguez, \& Diez, 1998). Cracking monitoring could play an important and effective role in evaluating structural health state. A wide variety of corrosion measurement techniques have been developed to analyze corrosion process and also to identify locations of corroded parts (Agarwala, Reed, \& Ahmad, 2000).

As novel techniques, optical fiber sensors exhibit many advantages. Compared to traditional sensors, they are flexible, embeddable, and electrical magnetic interference immutable. Many optical fiber sensors for corrosion monitoring have been reported. Optical fiber sensor with special polymer cladding has been invented (Ghandehari, 2001); it detects the change of cladding's properties caused by chloride corroding. The $\mathrm{pH}$ sensor used to monitor alkaline value of concrete is another kind of optical fiber sensor (Melhorn, Flachsbarth, \& Kowalsky, 2007). The optical fiber sensor by measuring light power reduction has been introduced (Leung, Wan, \& Chen, 2008); the decrease of iron film at the end of the fiber reduces light reflectivity from the fiber's end. However, the parameters measured by the aforementioned sensors are correlated to corrosion products or the environment; meanwhile the principles are rather complicated.

Monitoring of strain caused by corrosion would be the most straightforward and intelligible way to investigate corrosion. Pre-strained fiber Bragg grating (FBG) coated by metal tube has been developed (Lo \& Shaw, 1998), but corrosion of the metal tube itself would lead to misinterpretation of results. The improved FBG sensor is fabricated (Lee, Yun, \& Yoon, 2010); it is installed on a sacrificial plate, which is made of the same material as the structure. Although FBG sensors can get the corrosion strain, positions of reinforcement corrosion and concrete cracking are quite uncertain. It is impossible to consider all potential corrosion regions to install point-wise sensors like the FBG sensor.

The ability of the distributed monitoring of Brillouin Optical Time Domain Analysis (BOTDA) can overcome the above-said problems. In this paper, the theoretical model of cracking monitoring based on BOTDA is established. The optical fiber sensors are bonded around a cylindrical RC specimen to monitor the timedependent corrosion process. By analyzing the test results, the moment of initial cracking is judged and cracking width is estimated. 


\section{PRINCIPLE OF BOTDA}

The frequency shift has a linear relationship with strain and temperature. The relationship between strain variation $(\Delta \varepsilon)$, temperature change $(\Delta T)$, and Brillouin frequency $\operatorname{shift}\left(\Delta v_{B}(\varepsilon, T)\right)$ is shown in Equation (1) (Kwon, Kim, \& Choi, 2003):

$$
\Delta v_{\mathrm{B}}(\varepsilon, T)=\frac{d v_{\mathrm{B}}(T)}{d T} \Delta T+\frac{d v_{\mathrm{B}}(\varepsilon)}{d \varepsilon} \Delta \varepsilon
$$

There is no coupling between temperature and strain; therefore, temperature compensation fiber without strain can eliminate the influence of temperature on strain monitoring.

\section{TEST OF RUST EXPANSION SIMULATION}

\subsection{Test scheme}

To verify the feasibility of distributed optical fiber sensing technology in monitoring concrete cracking, we exerted circumferential stress to the inside holes of a concrete block, which can simulate the influence of steel corrosion to concrete, and what's more, distributed optical fiber sensors were applied to monitor the strain variations. The basic principle of the device can be shown as in what follows:

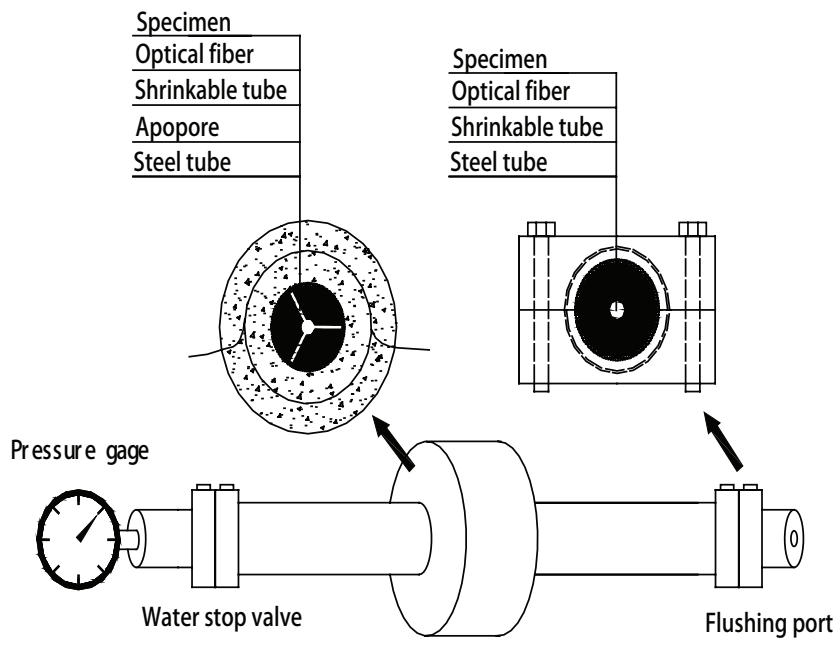

Figure 1. Schematic of internal pressure loading in concrete.

Water was added through the perforated pipes to produce circumferential stress, and the outer layers of the perforated steel pipes were covered with heat-shrinkable sleeve whose deformation and later pressure can be exerted to the inside holes directly. These above operations would finally lead to uniform circumferential stress acted on the specimen. Meanwhile, piezometers were used to measure the stress value, which can be applied as the circumferential stress exerted on the specimen. The water pressure can be added by booster pump. Repeated loading can bring the required pressure in the test. During the whole test, DITEST STA-R-type BOTDA sensors were used to monitor the optical fiber data when the specimen expanded.

The specimen size was set to an outer diameter of $75 \mathrm{~mm}$, and the length of the specimen surface fiber adhesion was kept on $2.0 \mathrm{~m}$, to eliminate the effects of spatial resolution. Holes were obligated in the middle of the specimen, whose diameter was kept the same as the latter accelerated corrosion diameter.

Plenty of preliminary tests show that specimens cast in the mixed proportion, as mentioned above, will crack when the internal pressure rises to about 6-7 MPa. If no restrictions were set on the outer surface of the specimen, the concrete will come up with apparent cracks immediately and remain in a precarious stage of development. Therefore, the test is carried out in two steps. First, we maintained the outer surface of the specimen free and load it in $1 \mathrm{MPa}$ load step to study the relationship between fiber strain and concrete expansion. When the pressure came to $6 \mathrm{MPa}$, we restricted the outer surface with fasteners. Under such operation, cracks cannot be seen until the pressure increased to about $10 \mathrm{MPa}$. Furthermore, reduce the internal pressure to $5 \mathrm{MPa}$ to observe the relationship between fiber strain and crack width. We control the crack development process by tightening the screw fasteners and use crack observation instrument to record the crack variation.

\subsection{Analysis of swelling and cracking}

DITEST STA-R type BOTDA was used in the optical fiber strain test, and the spatial resolution was set to $0.5 \mathrm{~m}$. The sampling point interval was $0.1 \mathrm{~m}$, and each load voltage level was kept exerting in $5 \mathrm{~min}$. We regarded the average value of three continuous data as the fiber strain under that level, and divided the whole fiber strain collecting process into two stages through the separatrix of crack appearance, as shown in what follows.

The above-mentioned curve shows that the maximum internal pressure the specimen can withstand before cracking is $10.0 \mathrm{MPa}$, and the limit cracking strain is around $150 \mu \varepsilon$. After cracking, the fiber strain sharply increased to as much as $300 \mu \varepsilon$. The cracking would exert intensive tensile strain to somewhere in the circumferential fiber, and what's more, release tensile strain generated by expansion. Thus, the fibers only suffered the tensile strain caused by the crack width variation. We controlled the crack width increasing by loosening the captive hoop. The above-mentioned process can only generate strain to the fibers arranged in cracks. 


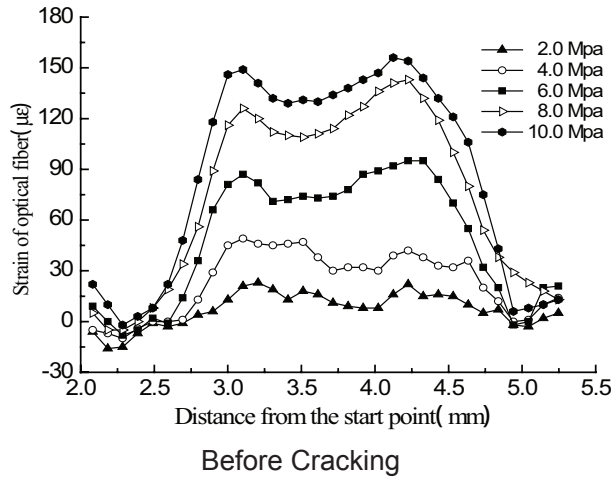

Figure 2. The fiber strain curve before and after cracking.

Maximum points of strain were chosen to analyze the cracking results. Fiber data under different load order were drawn as in what follows:

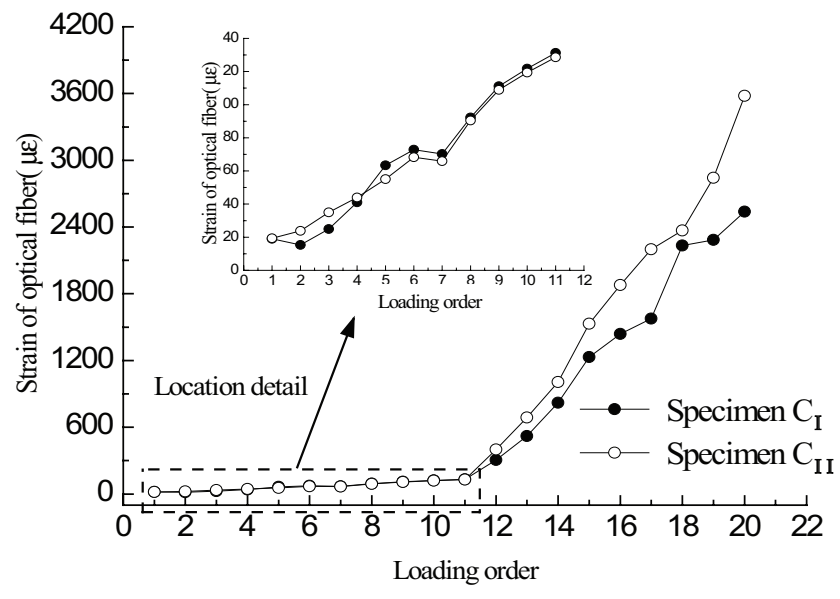

Figure 3. Strain curve of chosen sampling points.

From the above-mentioned curve, we can apparently get that the fiber data increased intensively at the 11th loading (10.0 MPa) and the specimen could be judged as cracking. The follow-up study will research the relationship between fiber strain and specimen expansion together with cracking.

\section{ACCELERATED CORROSION}

\subsection{Corrosion cracking detection}

Reinforcement corrosion is a time-dependent process. First, volume expansion of rust products creates pressure on boundary between concrete and steel bars and tensile strain around concrete is formed. Second, corrosion cracks are created on the surface of concrete once tensile strain has exceeded the ultimate tensile strain. Finally, cracking width increases as time passes. In this section, an accelerated corrosion test was applied to a cylinder specimen with $95 \mathrm{~mm}$ diameter to simulate reinforcement corrosion;

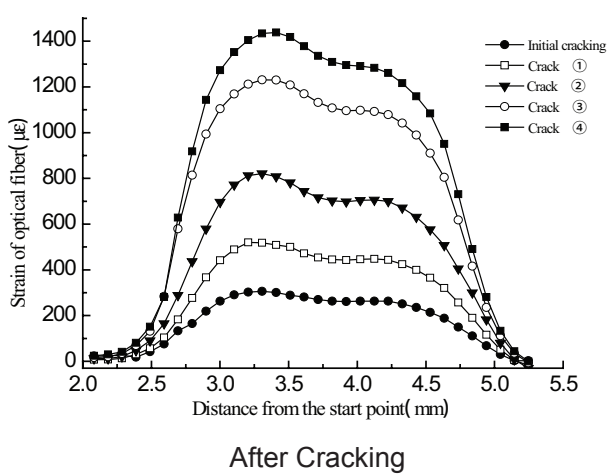

meanwhile, optical fiber sensors were used to measure cracking width.

\subsection{Design of accelerated corrosion test}

The steel bar with $16 \mathrm{~mm}$ diameter was connected to the positive pole of direct current, while a stainless steel net was tied on the negative pole. To accelerate corrosion processing, current density was kept at a high level $\left(0.5 \mathrm{~mA} / \mathrm{cm}^{2}\right)$ during the whole experiment. The specimen including connect fibers were immersed in $5 \% \mathrm{NaCl}$ saltwater. On one hand, it provided an environment for chloride corrosion. On the other hand, the temperature could keep constant at about $20^{\circ} \mathrm{C}$; therefore, the temperature affect could be ignored. All optical fiber sensors used the same material as calibration test, but two different kinds of bonding methods were used. As illustrated in Figure 4, one group of fiber sensors (P-1, P-2, P-3, and P-4) were bonded by point; therefore, the sensor length is $298 \mathrm{~mm}$ (close to $300 \mathrm{~mm}$ ); the other group of fiber sensors (D-1, D-2, D-3, and D-4) was adhered round the whole circle.

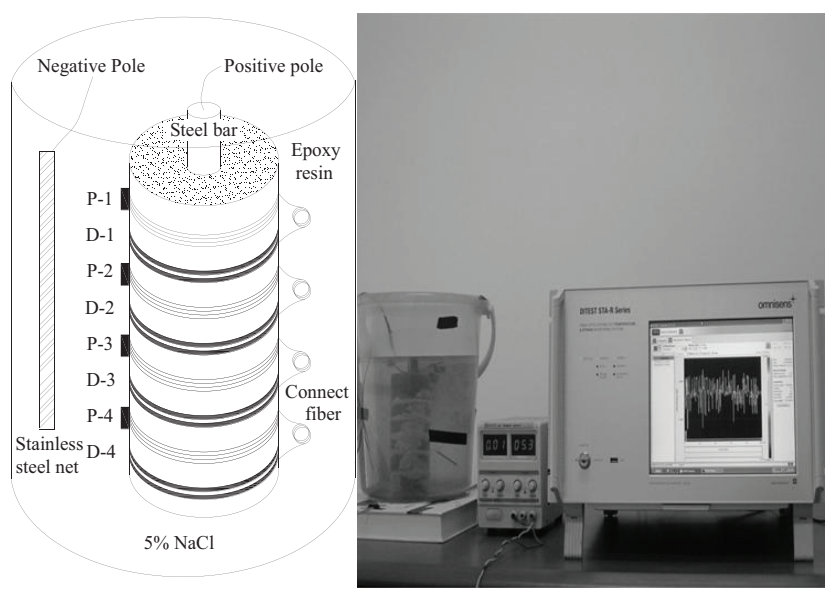

Figure 4. Layout of corrosion test.

All fibers were connected to strain measuring equipment BOTDA (DITEST STA-R Series), the $0.5 \mathrm{~m}$ spatial resolution and $0.1 \mathrm{~m}$ sample distance were 
set up. The corrosion experiment lasted for 3 days, all strain data were recorded by BOTDA automatically.

\subsection{Results and discussion}

Although the top of the specimen was airproofed by epoxy resin, a tiny bug between epoxy and concrete surface provided direct path for chloride migrating. As the experiment progressed, corrosion cracks appeared at the top of specimen first, then spread along the axial direction of the specimen. Figure 5 shows the final distribution of cracks; thicker dot dash lines present wider cracks.

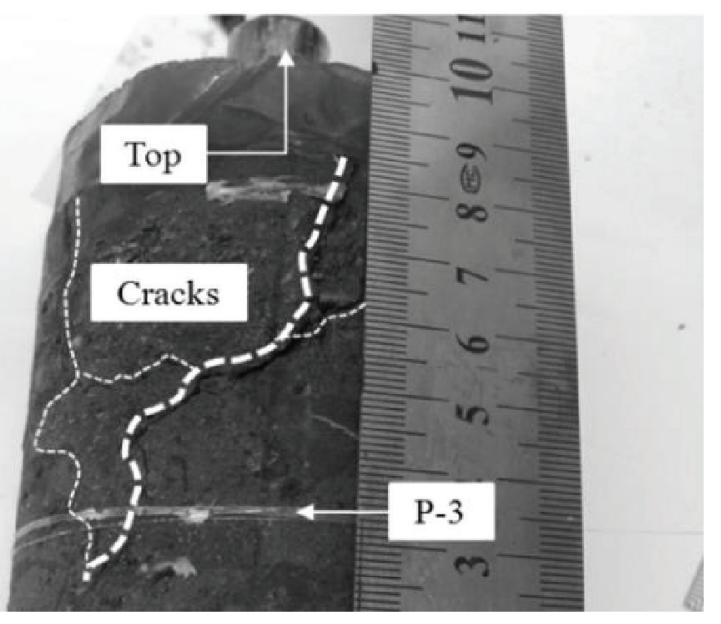

Figure 5. Final distribution of cracks.

Cracks were observed at five different sensing sections (P-1, D-1, P-2, D-2, and P-3). BOTDA can monitor and store fiber strain automatically; the entire expanding and cracking process was easily recorded, as shown as Figure 6. Fiber strains were plotted every $12 \mathrm{~h}$, five obvious sections with high tensile strain were observed.

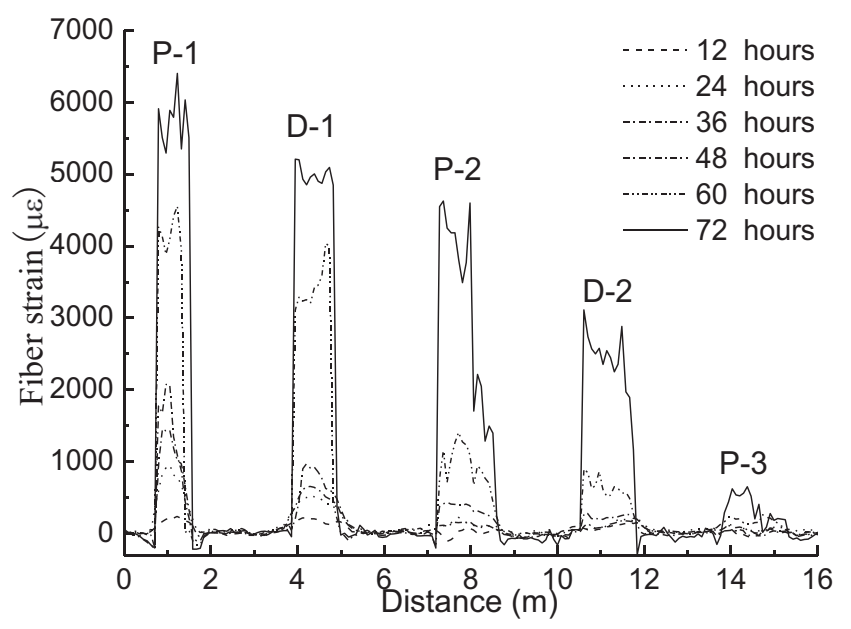

Figure 6. Fiber strain of the whole experiment.

It can be learned that strain of each section at the same time were different. Taking $\mathrm{P}-1$ and $\mathrm{P}-3$, for example, the maximum strain of $\mathrm{P}-1$ increased from 2000 to $6000 \mu \varepsilon$ at the third day (from 48 to $72 \mathrm{~h}$ ); however, strain of P-3 was only $700 \mu \varepsilon$ at $72 \mathrm{~h}$. By comparing strain values, it can be estimated that the cracking width in P-3 section could be 10 times smaller than section $\mathrm{P}-1$, which had been proved by visual inspection. In other words, the time-dependent characteristic of corrosion cracking can be achieved by comparing each sensing sections along the axial direction of the specimen. The obtained strain-time curve can reveal the steel corrosion process; however, the initial cracking time of concrete caused by steel corrosion needs further study.

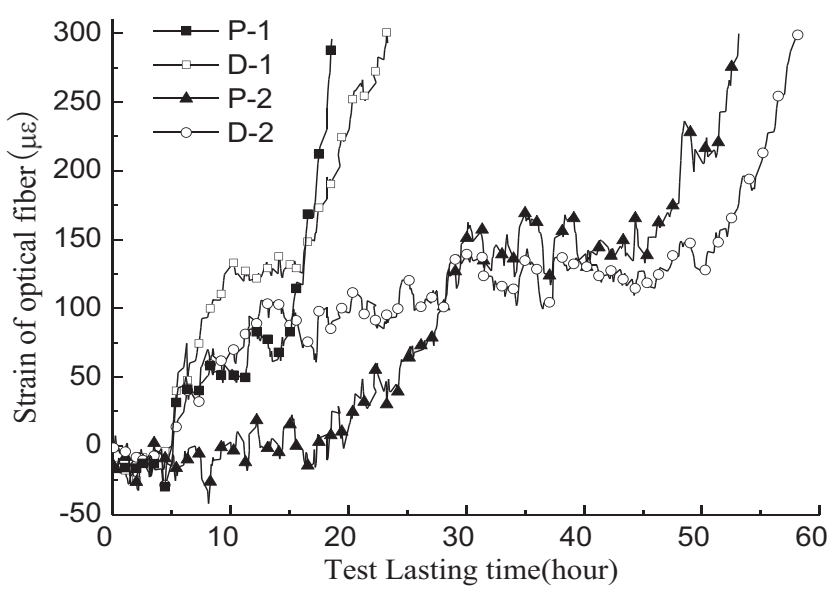

Figure 7. Four time curves of a sampling point in monitoring ring.

The earlier stage of steel corrosion only presents expansion rather than cracking. According to the results of pressurized simulation test, the sudden change of strain is the initial moment of specimen cracking. The above-mentioned curves reveal the process of steel corrosion expansion. The four curves show that based on the accelerated corrosion test, the subsequent strain appears rapidly increasing when the fiber strain reaches to $150 \mu \varepsilon$, and this moment of sudden change can be judged as the formation time of corrosion cracking. Such a phenomenon has been confirmed by manual observation. The reason for retaining fiber strain at around $150 \mu \varepsilon$ for a couple of times is mainly because of the process of corrosion products slowly filling the pores of the concrete, which takes some time. While the pressure simulation test contains no process of filling, the concrete specimen will crack immediately to the limit. Therefore, during the monitoring of reinforced concrete rust expansion and cracking by distributed optical fiber, certain time intervals should be set to do some early warning.

\section{CONCLUSION}

In this paper, the accelerated corrosion test was applied to the cylindrical specimen that had been installed 
with fiber sensor, and the strain of the specimen was recorded on-line by BOTDA. The moment of initial cracking was judged and also cracking width was estimated. This novel method was proved as a feasible and effective means to monitor reinforcement corrosion. However, further systematic research should be carried out to improve the stability, precision, and applicability of this method. The optical fiber that possesses the sufficient strength for visual inspection should be selected or fabricated. The relationship between fiber strain and cracking width also needs to be refined by more systemic experimental researches.

\section{ACKNOWLEDGMENT}

The project is supported by Natural Science Foundation of Ningbo (2013A610191), Natural Science Foundation of Zhejiang Province (Grant No. Q14E080022), and National Natural Science Foundation of China (51208459).

\section{REFERENCES}

Agarwala, V. S., Reed, P. L., \& Ahmad, S. S. (2000). Corrosion detection and monitoring - a review. Corrosion 2000, Houston, TX: NACE International. Alonso, C., Andrade, C., Rodriguez, J., \& Diez, J. M. (1998). Factors controlling cracking of concrete affected by reinforcement corrosion. Materials and Structures, 31(7), 435-441.

Ghandehari, M. (2001). Ingress monitoring in concrete structures. Proceedings of the 15th ASCE Conference on Engineering Mechanics, New York.

Kwon, I. B., Kim, C. Y., \& Choi, M. Y. (2003). Distributed strain and temperature measurement of a beam using fiber optic BOTDA sensor. Proceeding of SPIE, San Diego, USA, 486-496.

Lee, J. R., Yun, C. Y., \& Yoon, D. J. (2010). A structural corrosion-monitoring sensor based on a pair of prestrained fiber Bragg gratings. Measurement Science and Technology, 21(1), 1-7.

Leung, C. K. Y., Wan, K. T., \& Chen, L. (2008). A novel optical fiber sensor for steel corrosion in concrete structures. Sensors, 8(3), 1960-1976.

Lo, Y. L., \& Shaw, F. Y. (1998). Development of corrosion sensors using a single-pitch Bragg grating fiber with temperature compensations. Proceeding of SPIE, 3325, 64-72.

Melhorn, K., Flachsbarth, J., \& Kowalsky, W. (2007). Novel Sensors for long-term monitoring of $\mathrm{pH}$ and humidity in concrete. Proceeding of the 6th IWSHM Conference, Stanford, CA, 387-394.

Tuutti, K. (1982). Corrosion of steel in concrete. Swedish Cement and Concrete Research Institute, Stockholm. 